\title{
Determinants of Social Networking \\ Mechanisms and their Potential \\ Effects on a Place-Based \\ Geography: An Agent-Based \\ Simulation Approach
}

\author{
Andreas Koch \\ University of Salzburg, Austria
}

\begin{abstract}
The size and composition of social networks rely on the characteristics of the nodes and the infrastructure, both tangible and intangible. The networking mechanisms themselves, however, depend on circumstances such as meeting or team collaboration opportunities. A set of determinants of networking mechanisms will be discussed in this paper, and in particular the number of events over a given period is highlighted. This aim will be implemented by an agent-based simulation approach and empirically verified using network data from an Austrian regional project. Finally, the simulation results will be used to draw some conclusions on a place-based geography derived from the network-based geography.
\end{abstract}

\section{Keywords:}

team assembly mechanisms, event dependency, agent-based simulation, social networks

\section{Introduction}

There are many reasons why networks emerge among people. Sometimes the motivation is utilitarian, because people believe it is beneficial or profitable for them to collaborate. Sometimes an altruistic incentive might be the predominant cause for why they get connected, while in other cases it could be an exogenous (and involuntary) impetus that enforces cooperation. Thus, one's willingness and ability to connect with others appear to be a crucial issue in network creation.

There are further conditions that influence or even determine successful social networking. One of these, which we consider to be particularly relevant, is the number of events (workshops, meetings, etc.) offered to potential collaborators. Based on a theoretical reference model of team assembly mechanisms, an agent-based simulation model was developed that helps analyse determinants of social networking mechanisms. Node- and edge-based characteristics are correlated with an event-driven parameter and investigated within a certain timespan. Using the simulation results obtained through a comprehensive 
analysis of the parameter space, we look for patterns that allow us to draw conclusions from the relational network geography towards a place-based geo-spatial geography. The analysis is validated with empirical data obtained from two Austrian regions using a questionnaire conducted among team leaders of a social project.

\section{The Simulation of Networking Mechanisms}

The size and composition of social networks rely to a large part on the characteristics of the actors involved, i.e. their capabilities, needs and aspirations to collaborate with others. They also depend on the reasons for cooperation, the kinds of problems, and the (in)tangible infrastructure necessary to communicate via different channels. An important additional issue, however, comes with the nature of network mechanisms themselves - the process of how relations between actors emerge structurally and which determinants are thought to be relevant. This latter issue considerably affects the evolution of a large network that emerges from initially small teams and, by this, contributes to overcoming isolated and fragmented efforts towards a common goal. Achieving synergies through effective trans-local knowledge transfer is a goal that has been investigated in (social) network analysis for some time (Batagelj et al., 2014; de Nooy, Mrvar and Batagelj, 2005; Robins and Pattison, 2005). In this respect, agent-based simulation is an appropriate method to explore the underlying processes that lead to these networking mechanisms (Namatame and Chen, 2016).

As a first step of this investigation, we selected and then adapted to our own needs a theoretical reference model developed by Guimera et al. (2005) and made available as a NetLogo model by Bakshy and Wilensky (2007). The question raised by Guimera et al. (2005, p. 699) remains evident to some degree in our context too: 'Ts there a large connected cluster comprising most of the agents or is the network composed of numerous smaller clusters?' The ideal size does not necessarily correspond simply to exactly one allencompassing network: 'Successful teams evolve toward a size that is large enough to enable specialization and effective division of labor among teammates but small enough to avoid overwhelming costs of group coordination' (ibid., p. 697). Where our empirical research is concerned, we are less interested in economic costs than in the creation of sustainable social interactions.

Social interactions in collective human relations are commonly understood as a fundamental condition for human beings to live a satisfactory life. Establishing a personal identity and autonomy on the one hand, and trust, solidarity and commitment on the other hand require social-normative rules which function as a glue that helps agents to connect to one another. While the nature of social interaction is seen as an unquestioned fact, its valuation in operational terms is much harder to achieve (Koch, 2017). One apparently and generally accepted approach to operationalize the value of social interactions is given by the theory of social capital. A huge body of theoretical reflections as well as empirical studies consider transferring the concept of economic capital into the social realm as a suitable methodology for coping with social relations (see, for example, Field (2016) or Halpern (2005)). Within this context, we incorporate Bourdieu's (1986, p. 248) understanding of social capital as 'the aggregate of the actual or potential resources which are linked to possession of a durable 
network of more or less institutionalized relationships of mutual acquaintance and recognition'.

In this article, these assumptions are taken as relevant premises for networking mechanisms. In so doing, our focus shifts to the parameters that influence or determine the construction of large( $\mathrm{r}$ ) social networks that are initially small and more or less isolated due to the design of the social project. These parameters can be divided into three general domains: (i) actorbased parameters, (ii) linkage-based parameters, and (iii) place-based parameters. Sections 5 and 6 present some selected results.

\section{The Empirical Case Studies}

The empirical case studies for which an agent-based simulation model was constructed, which relies theoretically on the prototype model of Guimera et al. (2005), derive from an Austrian social project entitled 'Keep the Ball Rolling'. This project aims to enhance social well-being at a regional level by encouraging the local population to put into practice ideas that they are convinced are relevant. Individuals or small teams are called to submit project proposals that help reduce social injustice and promote social cohesion. Successful teams are awarded a grant to fund their projects, and in addition receive organizational support. The project started in the Lungau region (Salzburg) in 2011, and was taken up by Steirische Eisenstrasse (Styria), Mühlviertler Alm (Upper Austria), and Mostviertel Mitte (Lower Austria) regions (Gstach et al. 2015; Gstach et al. 2013). The empirical data used for the following simulation models is from the Styrian and Upper Austrian regions.

The project proposals are presented by the teams at three jury meetings. The successful candidates must implement their projects within 18 months. Every jury session is followed by a public celebration where the successful projects are presented. Because the implementation of a project is supported scientifically by a team of researchers for the duration of the project, several further meetings, ranging from small informal meetings to larger stakeholder workshops, are offered. Every team leader is invited to participate in a semi-standardized questionnaire designed to obtain knowledge about the project team in terms of its size and composition. The results of these questionnaires are used in the analysis which follows in Section 4.

The analysis is concerned with how the number of such meetings (i) determines the team assembly mechanisms, (ii) correlates with other determinants, and (iii) correlates with a placebased geography. This will be discussed in the following sections (5 and 6).

\section{The Model Design}

In order to analyse the development of collaborative socio-spatial network structures of initially small(er) and unconnected teams in two Austrian regions, a simulation model was constructed that includes the general ideas of the original model proposed by Guimera et al. (2005). Bakshy and Wilensky (2007) have made available a NetLogo version of the original model. This model serves as a reference model in that it helps understand the fundamental 
mechanisms of why and how project teams assemble over time. Hence, its first function is to provide a theoretical benchmark model that enables us to categorize the empirical regional models. This categorization was done by the author by an initial and preliminary statistical analysis (Koch, 2016), but will not be considered here.

Indeed, the reference model itself was derived from the original 'team assembly mechanisms' model and adapted to our own needs. There are a number of major differences between the reference model and the original model. (i) The number of teams is initialized at three time steps and not stepwise; with this adaptation, we represent the selection process of successful teams. (ii) Teams can vary in size. (iii) Teams can also vary in network structure; while the original model only allows the implementation of teams of three actors that are fully connected, the 'Keep-The-Ball-Rolling' project does not have such restrictions. (iv) Individual agents (and not only teams of three agents) are inserted as new potential collaborators; this feature will play a crucial role in the future modelling process when data sets are available that will be collected approximately two years after the official end of the social project (data for the Mühlviertler Alm region is currently missing, while data for the Steirische Eisenstrasse is not yet fully edited). We do not therefore refer to this characteristic of the model in this paper. (v) The original teams can be merged, either by a team leader (i.e. the leader of a project team), or by a team member (which is not possible in the reference model) who is selected stochastically for a new connection. This adaptation was implemented due to the nature of events that are realized during the different types of project meetings, which can consist of stakeholder workshops, project presentations and informal meetings, as well as ad hoc assistance from the staff of the regional offices. Although we do not consider these different event properties explicitly, they remain important to justify the 'connecting role' of both agent types, i.e. team leaders and team members. The original model takes only team leaders as connectors into account. Thus, the second function of the reference model is to serve as a prototype model from which tailored empirical models can be derived.

The construction of the two regional socio-spatial network models is based on standardized questionnaires that were conducted at the beginning of the project in each region. Every team leader of a project who was awarded a grant to participate with her/his project and who took part in the survey was asked about her/his team collaborators. We also know the team leader's home address (but not those of the collaborators, which affects the analysis). The numbers of actors and network ties are given in Table 1.

Table 1: Number of registered team leaders, team members, and network ties among and between them, in the Steirische Eisenstrasse (Styrian region) and the Mühlviertler Alm (Upper Austrian region).

\begin{tabular}{|l|l|l|l|}
\hline number of ... & team leaders & team members & network ties \\
\hline Styrian region & 79 & 396 & 441 \\
\hline Upper Austrian region & 59 & 418 & 655 \\
\hline
\end{tabular}

The two models are initialized with these settings of nodes and edges. In the style of the reference model, a six-dimensional parameter space is used to analyse the networking mechanisms among team leaders, team members, and between them. The aim of this 
procedure is to detect common patterns of relevance and relationships between the six parameters that seem to determine the behaviour of social networks in terms of their structure and dynamic. Table 2 gives an overview of the parameters and the ranges of values within which the further simulation results were analysed.

Table 2: Description of parameters used in the simulation models.

'values in round brackets indicate the increment value; for example: the parameter values for selAgents are 20,50 and 80

\begin{tabular}{|l|l|l|}
\hline parameter & description & $\begin{array}{l}\text { interval of } \\
\text { analysis }\end{array}$ \\
\hline selAgents & $\begin{array}{l}\text { probability of selecting agents per event who are willing } \\
\text { to collaborate }\end{array}$ & {$\left[20,(30)^{1}, 80\right] \%$} \\
\hline selAgentType & $\begin{array}{l}\text { probability of selecting team leaders or team members } \\
\text { per event who are willing to collaborate }\end{array}$ & {$[20,(30), 80] \%$} \\
\hline maxSelAgents & $\begin{array}{l}\text { potential maximum number of agents per event who are } \\
\text { willing to collaborate }\end{array}$ & {$[4,(4), 12]$ abs. } \\
\hline conTeamLeader & $\begin{array}{l}\text { probability of team leaders actually connecting with } \\
\text { other agents }\end{array}$ & {$[5,(15), 35] \%$} \\
\hline conTeamMember & $\begin{array}{l}\text { probability of team members actually connecting with } \\
\text { other agents }\end{array}$ & {$[0,(10), 30] \%$} \\
\hline numEvents & $\begin{array}{l}\text { number of events that enable the establishment of new } \\
\text { ties over a complete simulation run }\end{array}$ & $\begin{array}{l}{[1,2,4,8,16,32,} \\
40,80,160] \text { abs. }\end{array}$ \\
\hline
\end{tabular}

The parameter selAgents determines the likelihood that agents attending an event are willing to collaborate; for example, selAgents $=50 \%$ means that $50 \%$ of all attendees of a workshop are willing to collaborate. The parameter selAgentType specifies which agent type - the team leader or the team member - is more likely to be willing to collaborate. If selAgentType $=$ $50 \%$, both agent types are equally likely to be willing to collaborate; if selAgentType $<50 \%$, more team leaders are likely to be willing to collaborate. The interval of both parameters has a large range in order to detect network differences because of asymmetric probabilities. The maximum number of agents who are likely to collaborate, maxSelAgents, is a conservative estimate based on our experience of events we have organized in both regions. These three parameters affect the behaviour of the agents, while the following two affect the relationship between them. The conTeamLeader and conTeamMember parameters determine stochastically how agents connect among themselves. If, for example, conTeamLeader $=$ $10 \%$, then in $10 \%$ of all cases team leaders connect with other team leaders, and in $90 \%$ of all cases they connect with team members. The same rule applies to conTeamMember. The last parameter, numEvents, determines the number of events that take place during a simulation run. Events are defined here as face-to-face meetings and hence exclude Internetbased communications. The range of values varies between one event and 160 events. Taking into account that one simulation run takes 160 steps, which represents a time period of 160 weeks or almost three years (i.e., the project time of approx. 80 weeks and the postproject time of another 80 weeks when a posteriori questionnaires are conducted to evaluate 
the sustainability of the projects), the number of events varies between just one meeting during or after the social festival, and meetings on a weekly basis.

The social network simulation models were created using NetLogo 6.0 (Wilensky, 1999). The analysis of the modelling results has two stages: first, a statistical analysis composed of a multivariate linear regression analysis and a cluster analysis was conducted. Second, a simulation analysis consisting of representative simulation runs investigated the behaviour of the social networks by considering the network parameters 'closeness centrality' and 'betweenness centrality'. Figure 1 represents the simulation process graphically. The model is available as an updated version at OpenABM

(https://www.openabm.org/model/5583/version/1).

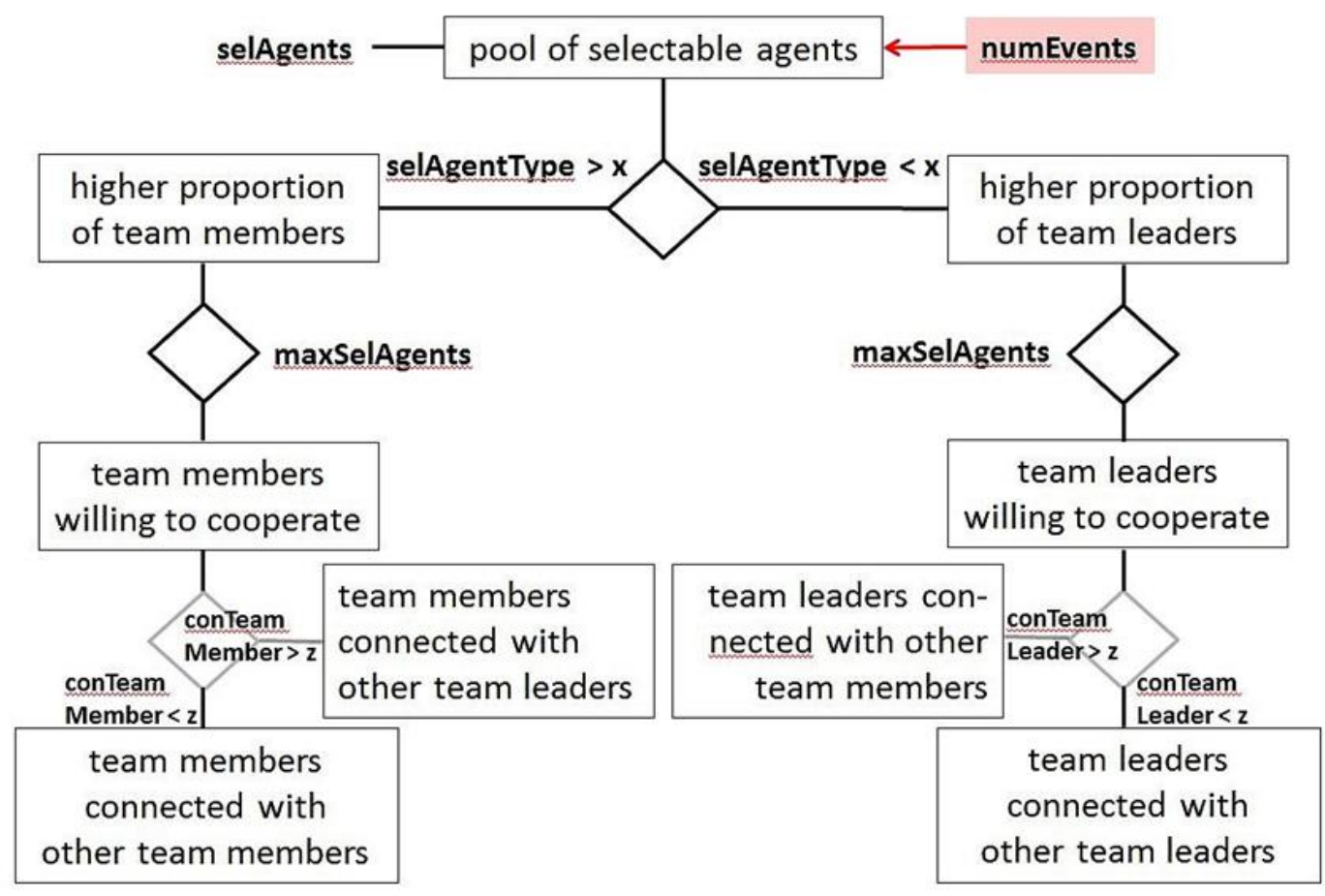

Figure 1: The flow diagram of the simulation model

\section{Some Selected Model Results}

Using the NetLogo extension of 'BehaviorSpace', all possible combinations of values (that is 3,888 in this case) within the intervals of the six variables were computed, which led to 3,888 mean values across all variations. These mean values were then used to compute average bivariate correlations and measurements of determination for the variables 'number of connections among team leaders', 'number of connections among team members' and 'number of connections between team leaders and team members'. The aim was to 
determine the strength and direction of relationships of the six independent variables by which the versatile network structures can be explained. These different network structures consist of both the initial network relations of the original teams (empirical data) and the network relations created during the simulation (modelled data). The focus - compared with the original team assembly model - is only on the development of links, since no new agents were introduced.

The method used to create multiple regressions was 'stepwise selection', which avoids multicollinearity to some degree. Table 3 reveals that numEvents is the most relevant independent variable for all three dependent variables. The measurement of determination (R2) confirms this statement. The parameter numEvents is, however, more relevant to explain the variation of the distribution of the links among team leaders than among team members. On average, team leaders benefit more than team members from an increase in the number of workshops and meetings. This is due in part to an implicit bias, occurring because there are fewer team leaders than team members, which quickly leads to a higher number of linkages. This assessment is confirmed by the contrary fact of there being only a few events ( 1 to 8$)$ : under this condition, team leaders are far more involved than team members in creating a large connected social network. This fact has to be taken into account to avoid lock-in effects of a well-informed stakeholder group.

The statistical influence of the remaining five parameters is significantly less relevant in explaining the variation of ties among and between the two groups when compared with numEvents, as is illustrated in the last column of Table 3. The least relevant parameter in terms of correlation is conTeamMember for the group of team leaders, and conTeamLeader for the group of team members. One conclusion might be that even a low chance of actually getting connected is sufficient if there are a high number of opportunities to meet others.

Table 3: Regression patterns of the three dependent edge-related variables for the Steirische Eisenstrasse (Styria) and the Mühlviertler Alm (Upper Austria).

\begin{tabular}{|l|l|l|l|}
\hline dependent variable & $\begin{array}{l}\text { most relevant } \\
\text { independent } \\
\text { variable }\end{array}$ & $\begin{array}{l}\mathbf{R}^{2} \text { of most relevant } \\
\text { independent } \\
\text { variable }\end{array}$ & $\begin{array}{l}\mathbf{R}^{2} \text { of all included } \\
\text { independent } \\
\text { variables }\end{array}$ \\
\hline $\begin{array}{l}\text { number of connections } \\
\text { among team leaders } \\
\text { (Styria) }\end{array}$ & $\begin{array}{l}\text { number of events } \\
r=0.785\end{array}$ & 0.617 & 0.759 \\
\hline $\begin{array}{l}\text { number of connections } \\
\text { among team leaders } \\
\text { (Upper Austria) }\end{array}$ & $\begin{array}{l}\text { number of events } \\
r=0.779\end{array}$ & 0.606 & 0.751 \\
\hline $\begin{array}{l}\text { number of connections } \\
\text { among team members } \\
\text { (Styria) }\end{array}$ & $\begin{array}{l}\text { number of events } \\
r=0.633\end{array}$ & 0.401 & 0.525 \\
\hline $\begin{array}{l}\text { number of connections } \\
\text { among team members } \\
\text { (Upper Austria) }\end{array}$ & $\begin{array}{l}\text { number of events } \\
r=0.623\end{array}$ & 0.388 & 0.505 \\
\hline $\begin{array}{l}\text { number of connections } \\
\text { among all actors (Styria) }\end{array}$ & $\begin{array}{l}\text { number of events } \\
r=0.756\end{array}$ & 0.572 & 0.687 \\
\hline
\end{tabular}




\begin{tabular}{|l|l|l|l|}
\hline $\begin{array}{l}\text { number of connections } \\
\text { among all actors (Upper } \\
\text { Austria) }\end{array}$ & $\begin{array}{l}\text { number of events } \\
r=0.735\end{array}$ & 0.540 & 0.652 \\
\hline
\end{tabular}

The cluster analysis aims to explore further structures that have been unknown so far. The cluster algorithm used here is the "Ward method", which yields more or less evenly distributed clusters. A variation of four to six clusters was applied, and the solution with five clusters provided good results with respect to a good discrimination of the values and interpretation of the results. As Tables 4 and 5 illustrate for both regions in a very similar way, the highest numbers of connected agents (cluster 5 in both cases) are achieved when numEvents is highest (which is not surprising), the likelihood of selected agents is relatively high, the proportion of team leaders is higher than that of team members, the maximum number of potentially selectable agents is relatively high, and the likelihood that agents will establish ties is high. If more team members are likely to be selected (selAgentType $>50 \%$ ), then a considerable decrease of realized linkages follows. Again, the least relevant parameters are con'TeamLeader and con'TeamMember (although they are responsible for the relevant discrimination between cluster 4 and cluster 5 in the Upper Austrian case study).

Table 4: Cluster Analysis results for the Styrian case study. Values represent mean values.

\begin{tabular}{|l|l|l|l|l|l|}
\hline dependent variables & Cluster 1 & Cluster 2 & Cluster 3 & Cluster 4 & Cluster 5 \\
\hline number of ties among team leaders & 700 & 1,041 & 156 & 1,263 & 1,415 \\
\hline number of ties among team members & 805 & 2,075 & 83 & 3,402 & 9,074 \\
\hline number of all ties & 2,132 & 4,248 & 497 & 7,824 & 10,423 \\
\hline & & & & & \\
\hline independent variables & & & & & \\
\hline numEvents & 73 & 118 & 18 & 141 & 160 \\
\hline selAgents & 58 & 58 & 47 & 70 & 73 \\
\hline selAgentType & 52 & 50 & 51 & 40 & 32 \\
\hline maxSelAgents & 7 & 7 & 6 & 8 & 8 \\
\hline conTeamLeader/conTeamMember & $20 / 15$ & $20 / 15$ & $20 / 15$ & $19 / 13$ & $20 / 25$ \\
\hline
\end{tabular}

Table 5: Cluster Analysis results for the Upper Austrian case study. Values represent mean values.

\begin{tabular}{|l|l|l|l|l|l|}
\hline dependent variables & Cluster 1 & Cluster 2 & Cluster 3 & Cluster 4 & Cluster 5 \\
\hline number of ties among team leaders & 184 & 932 & 1,004 & 1,299 & 1,364 \\
\hline number of ties among team members & 141 & 1,458 & 5,293 & 2,682 & 10,459 \\
\hline number of all ties & 817 & 4,056 & 7,229 & 12,152 & 12,844 \\
\hline & & & & & \\
\hline independent variables & & & & & \\
\hline numEvents & 22 & 104 & 133 & 155 & 160 \\
\hline selAgents & 47 & 60 & 66 & 70 & 72 \\
\hline selAgentType & 51 & 51 & 38 & 34 & 34 \\
\hline maxSelAgents & 6 & 7 & 7 & 9 & 9 \\
\hline conTeamLeader/conTeamMember & $20 / 15$ & $20 / 13$ & $20 / 22$ & $20 / 4$ & $21 / 25$ \\
\hline
\end{tabular}


Although numEvents appears to be a highly relevant determinant in the development of large network compositions, it is also highlighted in the cluster analysis results that team members benefit significantly from an increase in the number of events. A comparison of cluster 1 and cluster 2 for the Steirische Eisenstrasse reveals that almost doubling numEvents leads to a considerably higher increase of tied team members (approx. 2,100 compared with 800) than of tied team leaders (approx. 1,500 compared with 700).

A simple concentration measurement that takes the relative development of linkages into account reveals another considerable difference between team leaders and team members. For team members, the highest decile comprises $5 \%$ to $100 \%$ of all realized connections with other agents - i.e., $90 \%$ of cases represent only up to $4 \%$ of all connections. For team leaders, however, the highest decile comprises a minimum of $57 \%$ of all realized connections. These ratios of concentrations are true for both case study regions.

In addition to the statistical analysis, a simulation analysis was performed in order to investigate the process of the network creation. For this purpose, two common centrality measures were used, namely the closeness centrality and the betweenness centrality. Both centrality measures characterize an agent's position or role in the entire network. In NetLogo, closeness centrality is defined as 'the inverse of the average of an [agent's] distances to all other [agents]' (Wilensky, 1999, n.p.). Distances are defined as shortest paths. Betweenness centrality, by contrast, refers to the mediator function of an agent (for example, mediating communication flows). To calculate the betweenness centrality of an agent, 'you take every other possible pairs of [agents] and, for each pair, you calculate the proportion of shortest paths between members of the pair that passes through the current [agent]. The betweenness centrality of an [agent] is the sum of these' (Wilensky, 1999, n.p.).

Based on typical and representative simulation runs of the above-mentioned behaviour space analysis in NetLogo, a data subset with 32 cases was extracted. Extraction here means that extreme values of parameters were excluded; for example, numEvents was set to 80 in one case and to 8 in another. Figure 2 illustrates the results of agents that have a high closeness centrality ('high' defined as above the threshold value of 0.5) for the Steirische Eisenstrasse region. The two maps differ in the number of events during the simulation run.
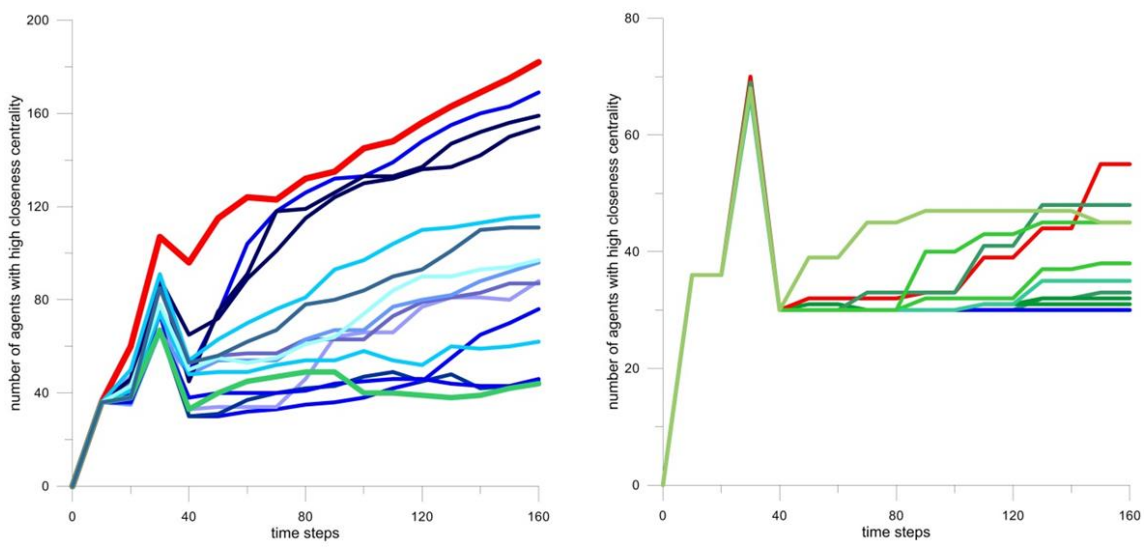

Figure 2: Distribution of high closeness centrality agents for numEvents $=80$ (left) and numEvents $=8$ (right) in the Styrian study area 
16 out of the 32 simulation runs were executed using a high number of meeting events (lefthand side), while the other 16 used a low number of events (right-hand side). When we compare the two graphs of Figure 2, the most obvious fact is that the variation of results with numEvents $=80$ is considerably larger than with numEvents $=8$. The case representing the lowest number of agents gaining a high closeness centrality (green graph in the left diagram) is characterized by a high number of selected team leaders (because selAgents is high and selAgent'Type is significantly below the 50\% threshold value) and by high linkage percentages for both agent types (see Table 6). The case with the highest number of agents gaining a high closeness centrality (red graph in the left-hand diagram) differs in the relationship of selAgents and selAgentType, which is now exactly the opposite. These results highlight the important contribution of team leaders in two ways: (i) they are important as central nodes within the social network; (ii) they also act as multipliers for team members to achieve a central position in the network.

Table 6: Parameter settings that lead to the 'highest number' and 'lowest number' of agents with a high closeness centrality for numEvents $=80$ and numEvents $=8$, respectively. Values are from the Styrian study area.

\begin{tabular}{|l|l|l|l|l|}
\hline numEvents $=80$ & selAgents & selAgentType & conTeamLeader & conTeamMember \\
\hline highest number & 80 & 20 & 35 & 30 \\
\hline lowest number & 20 & 80 & 35 & 30 \\
\hline numEvents = 8 & selAgents & selAgentType & conTeamLeader & conTeamMember \\
\hline highest number & 80 & 20 & 35 & 1 \\
\hline lowest number & 20 & 20 & 35 & 30 \\
\hline
\end{tabular}

Fluctuations for cases with numEvents $=8$ (Figure 2, right-hand diagram) are far less significant. Here, values range from 30 (blue graph) to 55 (red graph) (there are six different combinations of parameter values that lead to 30 agents gaining high closeness centrality). The most relevant determinants to explain the differences are selAgents and conTeamMember - one node-related and one linkage-related parameter. A comparison of the two diagrams of Figure 2 convincingly shows that even a high number of events supplied do not guarantee a sufficiently high number of agents who are tightly linked together if the other parameter values do not foster successful collaboration efforts.

\section{Possible Impacts of Network Configurations on a Place-Based Geography}

The statistical and simulation analyses reveal that the construction and (sustainable) consolidation of social networks are influenced by a high number of factors whose interrelationships are quite complicated in terms of generating a large(r) connected network. Offering a high number of events does not automatically ensure that a high proportion of agents will gain high centrality in order to provide for efficient knowledge transfer within the social network (Koch, 2017). In fact, even only a few events can result in a reliable number of durable linkages among agents. The results emphasize that agents' willingness and 
opportunities to collaborate can have both a cushioning and an amplifying effect on knowledge dissemination within the networks.

However, one must take the specific sequence of the three jury meetings into account. Due to the inclusion of new team leaders and team members at predefined time steps, a temporary decline in the number of agents with high betweenness and/or closeness centrality can arise. Fluctuations are large $(r)$ if the number of events provided is high, because meetings are likely to take place between jury sessions, too.

Therefore the organization of events to foster collaboration is a challenging undertaking, as social network analysis has shown. The supply of opportunities to meet each other in order to exchange knowledge and experience has, in addition to its qualitative component (obligatory stakeholder meeting vs. informal team meeting), a quantitative tendency. Setting aside all the network-based determinants discussed so far, one should not forget the geographical domain - i.e., the geospatial distribution of the relevant actors. In other words, a translation from a space-of-flows geography to a space-of-places geography seems appropriate.

Figures 3 and 4 are representative extracts of the spatial distribution of agents with high betweenness centrality (yellow) and high closeness centrality (violet), and remaining team leaders (red) for a high and low numbers of events (numEvents $=80$ and numEvents $=8$ respectively). Other parameter values are close to those in Table 6, and linkages are hidden. The scale of resolution of the agents' locations, which is based on the questionnaire, is the municipal level. Distribution within the municipalities is for visualization purposes only.
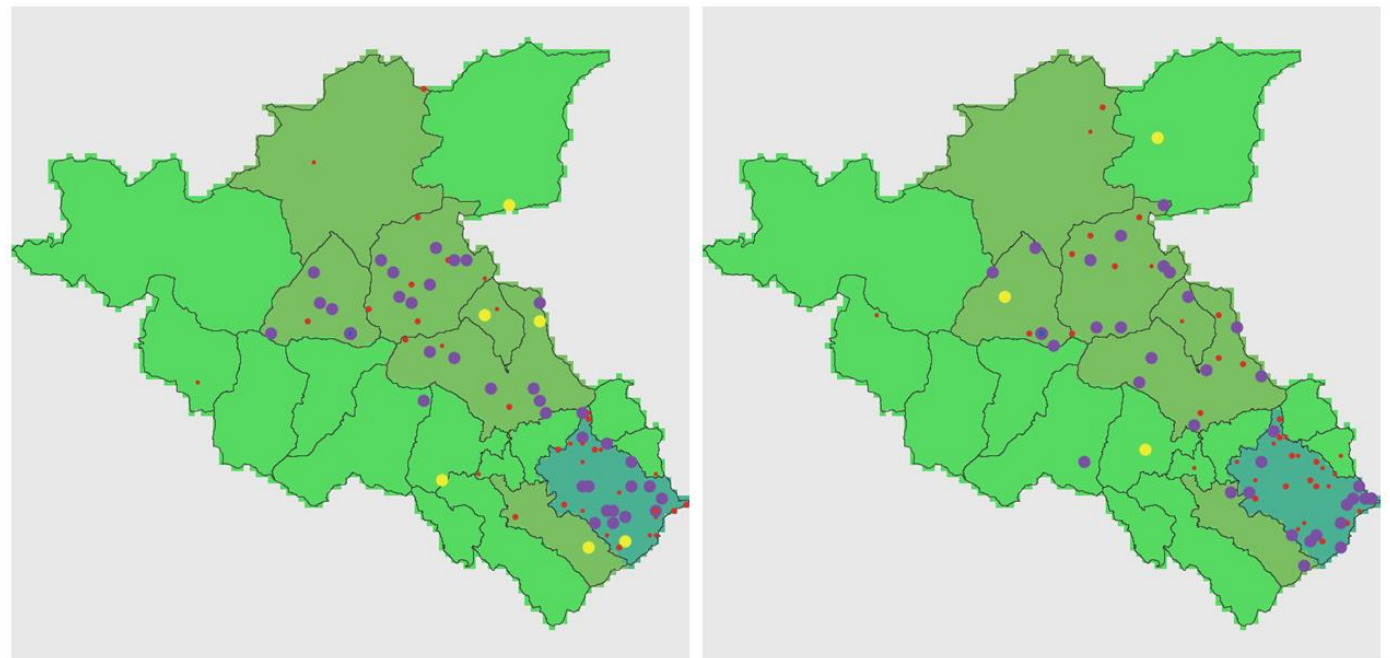

Figure 3: Distribution of agents with high betweenness centrality (yellow) and high closeness centrality (violet) for numEvents $=80$ (left) and for numEvents $=8$ (right) in the Steirische Eisen- strasse. Colours of municipalities indicate less than $2 \%$ of all projects (light green); between $2 \%$ and $20 \%$ (green); more than $20 \%$ (dark green). 

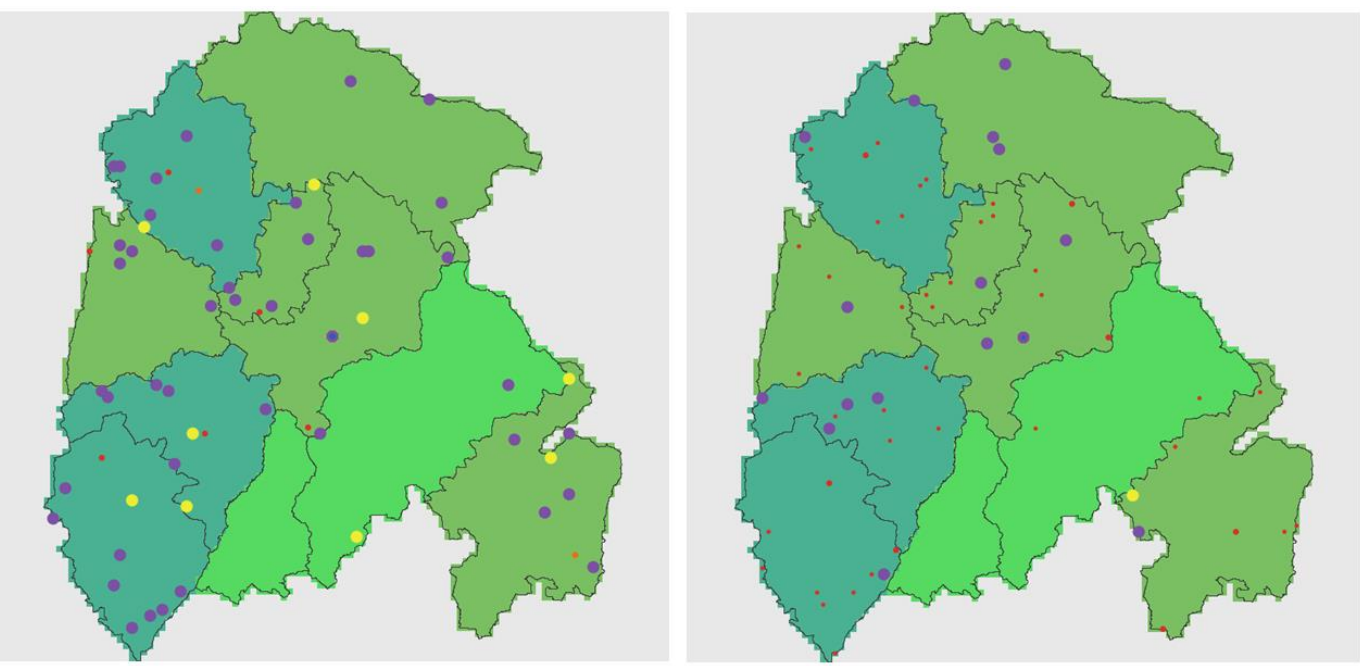

Figure 4: Distribution of agents with high betweenness centrality (yellow) and high closeness centrality (violet) for numEvents $=80$ (left) and for numEvents $=8$ (right) in the Mühlviertler Alm region. Colours of municipalities indicate: less than $5 \%$ of all projects (light green); between $5 \%$ and $15 \%$ (green); more than $15 \%$ (dark green).

One important conclusion that can be drawn from the distribution patterns is that the vertical structures of the social networks do not completely coincide with the structure of the places where the team leaders reside and where the projects were implemented. The initial network pattern is characterized by many small and largely unconnected teams each with one leader and a couple of members who are, of necessity, linked to the leader. This vertical structure was then reiterated as team leaders connected to one another or to other team members. These linkage mechanisms determine the simulated structural pattern that correlates hypothetically with the similar, project-related aspirations of the other actors involved (research team, purchaser and regional office staff). Agents with high closeness and/or betweenness centrality are disproportionately more often located in municipalities with comparatively fewer implemented projects (light green areas). This is true for both situations with a high number of events and those with a low number. If a high number of meetings were to be offered (maps at the left-hand side), then the distribution of agents with high betweenness centrality ( $=$ important communicators) would be more even than the distribution of the projects. The result is true for the Styrian as well as the Upper Austrian study areas. In fact, this statement can be extended to the situation of a low number of offered meetings if the closeness centrality ( $=$ strong ties between agents) is taken into consideration, as can be seen in the maps at the right-hand side of Figures 3 and 4. The peculiar relationship between the two geographies immediately prompts the conclusion that the decisions about adequate venues for meetings should be made by taking the whole project region into account and not concentrating mostly on the region's larger towns.

Another conclusion that can be drawn is that with a more even spatial distribution of highly centralized agents, a proper coverage of network geography and place-based geography can be achieved in terms of communications (space of flows) and localized decisions (space of 
places). If this is true, then local projects can benefit from each other thanks to this type of knowledge dissemination.

Ultimately, personal engagement in one's own local social environment also needs to be appreciated by rotating meeting locations across the entire region, because then 'peripherally located' agents can act as hosts and can proudly present their project work in immediately visible form.

\section{Conclusions}

If we recall the project implementation process - people submit a project proposal at a series of three jury meetings, the selection of good projects is independent of their location, meetings offer the opportunity to collaborate -, then we can conclude that the number of events has some impact on the creation of highly centralized network agents, who represent important mediators and communicators, i.e. nodes that keep the large(r) connected network together. Simulation outcomes illustrate that it is possible to translate the more or less even distribution of centralized agents within the networks into a more or less even geographical distribution of them. One determining factor, then, would be to geographically distribute event locations adequately. This assumption can be empirically validated in both study regions since the organization of events took into account explicitly the idea of a geospatially wide distribution of events.

The current model considers different types of events only implicitly, by the number of events: a biweekly meeting frequency will include informal small meetings, while eight meetings over three years are likely to be large formalized meetings. This quantitative differentiation must be improved by developing our model to include weights. A second improvement would be the implementation of a model procedure which allows for the disconnection of agents during the simulation based on the intensity of their relations; a first attempt to formulate such a model is outlined in Koch (2017).

\section{References}

Bakshy, E. \& Wilensky, U. (2007): NetLogo Team Assembly model. Center for Connected Learning and Computer-Based Modeling, Northwestern University, Evanston, IL. Retrieved from http://ccl.northwestern.edu/ netlogo/models/TeamAssembly.

Batagelj, V., Doreian, P., Ferligoj, A., \& Kejžar, N. (2014): Understanding Large Temporal Networks and Spatial Networks. Chichester, John Wiley \& Sons.

Bourdieu, P. (1986): The forms of capital. In: Richardson J. (Ed.): Handbook of Theory and Research for the Sociology of Education (translated by Richard Nice). New York, 241-258, Retrieved from https://www.marxists.org/reference/subject/philosophy/works/fr/bourdieu-forms-capital.htm

de Nooy, W., Mrvar, A., \& Batagelj, V. (2005): Exploratory Social Network Analysis with Pajek.

Cambridge, Cambridge University Press.

Field, J. (2016). Social Capital. 3rd edition, New York: Routledge.

Gstach, I., Kapferer, E., Koch, A., \& Sedmak, C. (eds.) (2013): Sozialatlas Steirische Eisenstraße.

Wien, Mandelbaum Verlag. 
Gstach, I., Kapferer, E., Koch, A., \& Sedmak, C. (eds.) (2015): Sozialatlas Mühlviertler Alm. Wien, Mandelbaum Verlag.

Guimera, R., Uzzi, B., Spiro, J., \& Amaral, L.A.N. (2005): Team Assembly Mechanisms Determine Collaboration Network Structure and Team Performance. Science, Vol. 308, 29 April 2005, 697702.

Halpern, D. (2005). Social Capital. Cambridge: Polity Press.

Koch, A. (2016): The Impact of Event Determinants on Team Assembly Mechanisms of Social Networks. ESSA Proceedings 2016, Rome.

Koch, A. (2017): A Simulation Model Perspective on Social Capital. ESSA proceedings 2017, Dublin. (in print).

Namatame, A., \& Chen, S.-H. (2016): Agent-Based Modeling and Network Dynamics. Oxford, Oxford University Press.

Robins, G., \& Pattison, P. (2005): Interdependencies and Social Processes: Dependence Graphs and Generalized Dependence Structures. In: Carrington P. J., Scott J., Wasserman S. (eds.): Models and Methods in Social Network Analysis. Cambridge, Cambridge University Press.

Wilensky, U. (1999): NetLogo. Center for Connected Learning and Computer-Based Modeling, Northwestern University, Evanston, IL. Retrieved from http://ccl.northwestern.edu/netlogo/. 